\title{
8 Paläoanthropologie und Archäologie: Inferenzen zu kognitiver Modernität
}

\begin{abstract}
Language affords culture-carrying capacity (e.g. there are no advanced technologies without language), and this linkage allows reasonable inferences from the archeological record. Therefore, we think it is overwhelmingly likely that Neanderthals were as much articulate beings as we ourselves are, that is, with large vocabularies and combinatorial structures that allowed propositional content and illocutionary force to be conveyed. Only such an advanced communication system could have carried the advanced cultural adaptations that Neanderthals exhibited. However, some of the new findings, especially those coming from the genetics of brain development and neurocognitive disorders, seem to strongly suggest that, despite a profound shared humanity, archaic and modern humans were not identical.
\end{abstract}

Dediu \& Levinson (2018: 52)

\subsection{Zum Mehrwert der vorliegenden Analyse}

Im Grunde stellen Dediu \& Levinson mit ihren Aussagen pointiert fest, worauf die jüngeren Ergebnisse der Paläoanthropologie, der Archäologie sowie der Paläogenetik allesamt - im Kontrast zu klassischen Interpretationen - hinzuweisen scheinen. In dem Versuch, deren zusammenfassenden Ausführungen Wertiges hinzustellen zu können, motiviert sich der vorliegende Abschnitt auf zweierlei Weise. Erstens fußen deren Schlussfolgerungen primär auf dem direkten Vergleich zwischen dem Neandertaler und dem anatomisch modernen Menschen hinsichtlich fossiler Funde und ausgewählter genetischer Erkenntnisse sowie der darauf aufbauenden Ableitung von Verhaltensweisen, kognitiver Leistungsfähigkeit und dem Vorhandensein von Kultur sowie Sprache. Dieses Vorgehen konstituiert durchaus ein umfassendes, zusammenfassendes Programm, jedoch kann dieses Bild zusätzlich erweitert werden. Der damit zu erreichende, vergrößerte Gesamtkontext vermag es, die Validität jener Schlussfolgerungen zusätzlich zu steigern und in Folge mit vergrößertem Nachdruck nahezulegen.

Zweitens kann ein zusätzlicher analytischer Mehrwert dadurch geschaffen werden, dass die historische Herausbildung der bisherigen, zunehmend veralteten Lehrmeinung skizziert wird. Ebendiese klassische Lehrmeinung postuliert, dass der anatomisch moderne Mensch dem Neandertaler hinsichtlich seiner Kognition überlegen gewesen sei und folglich hinsichtlich seines Verhaltens, seiner Technologien, seiner Kultur und in letzter Konsequenz seiner sprachlichen Befähigung eine größere qualitative und quantitative Leistungsfähigkeit 
besessen habe. Nicht zuletzt wird die Ersetzung des Neandertalers durch den modernen Menschen, die vor grob 40.000 Jahren stattfand, mittels Verweises auf dieses Ungleichgewicht mit scheinbar großer Plausibilität erklärt.

Das vorliegende Kapitel beschreibt zu diesem Zweck zunächst ebendiesen Weg zur klassischen, inzwischen wenigstens teilweise veralteten Position zum Verhältnis zwischen dem Neandertaler und dem anatomisch modernen Menschen, skizziert darauf aufbauend mehrere jüngere Erkenntnisse sowie Argumentationslinien und verbindet den sich dazu aktuell herausbildenden Konsens der einschlägigen Forschung mit weiteren, bei Dediu \& Levinson (2018) unerwähnten Erkenntnissen in einer übergreifenden Synthese. Das Gesamtbild, welches sich hieraus ergibt, gesellt sich zu deren Interpretation in unterstützender Weise und suggeriert einen vergleichsweise alten kumulativen kulturellen Komplex, der mit nennenswerter Wahrscheinlichkeit auch eine leistungsfähige (proto)sprachliche Befähigung impliziert. Dies steht freilich im Kontrast zu Autoren, welche aus motivierten Gründen an der klassischen Interpretation festhalten (vgl. bspw. die Tradition von Berwick, Hauser \& Tattersall 2013 sowie Berwick \& Chomsky 2016 und 2017).

\subsection{Die historische Entwicklung zur klassischen Auslegung}

Für ein analytisch leistungsfähiges Verständnis zum Forschungsverlauf, welcher erst jüngst in Richtung einer Anerkennung hoher kognitiver Fähigkeiten beim Neandertaler verläuft, ist eine Klärung der Forschungsgeschichte von großer Bereicherung. Um jedoch den eigentlichen Argumenten und den jüngeren Daten ausreichend Raum zu bieten, soll diese Klärung in zweckmäßig knapper Form geschehen. Es soll daher lediglich festgestellt werden, dass trotz Funden wie dem des „Java-Menschen“ (Dubois 1894) die Paläoanthropologie im Verlauf des 19. Jahrhunderts umfänglich von der europäischen Fundlage dominiert wurde und dass sich diese Situation bis weit in das 20. Jahrhundert hinein aufrechterhielt. Dies begründet sich schon bereits darin eingängig und umfassend, dass in Europa Jahrhunderte der Industrialisierung, der Schaffung von Infrastruktur, des Abbaus von Bodenschätzen und dergleichen in großem Umfang archäologische und paläoanthropologische Funde zutage förderten. Eine zusätzliche Rolle spielte hier sicherlich auch die relativ große Bevölkerungsdichte Europas und nicht zuletzt lag das Zentrum der sich zunehmend herausbildenden und wachsenden Naturwissenschaften für lange Zeit auf primär eu- 
ropäischem Boden. Die gesamte wissenschaftliche Infrastruktur war damit in mehrerlei Hinsicht fokussiert - in Europa und auf Europa. ${ }^{39}$

Auch als sich afrikanische Funde aufgrund eines wachsenden Forschungsinteresses und im Kontext besserer Technologien sowie einem wachsenden Wissen darum, auf welche Weise und in welchen Regionen nach Funden zu suchen ist, schließlich mehrten, hielt sich weiterhin ein starkes Ungleichgewicht, welches trotz einer sukzessiven Rückbildung ebendieses Ungleichgewichts bis heute $\mathrm{zu}$ beobachten ist. So stellt Hublin (Interviewzitat aus Bahnsen 2014) fest: „Über Europa und Asien wissen wir viel mehr als über den Ursprungskontinent der Menschheit. Ausgerechnet Afrika ist unser größtes Rätsel.“ In diesem Sinne geschah die Rekonstruktion des anatomisch modernen Menschen in physiologischer, kognitiver, kultureller und technologischer Hinsicht umfassend über europäische Funde, welche schlicht den Großteil der Datenlage lieferten. In Folge entstand eine empirisch fundierende Plattform, auf welcher problematische Fehlschlüsse aufbauen. Für weitere Belege zu diesen letzten Punkten vergleiche auch Grayson (1983) sowie Gamble (1999).

Wie im einführenden Kapitel zur Menschwerdung bereits angesprochen wurde, ersetzte der anatomisch moderne Mensch den Neandertaler vor grob 40.000 Jahren (vgl. bspw. Benazzi et al. 2011; Higham et al. 2014) in einer noch nicht vollständig geklärten Dynamik. ${ }^{40}$ Die fossile Datenlage lieferte dabei ein Bild, in dem zeitlich vor dieser Ersetzung eine technologisch einfachere Neandertaler-Population in Europa vorherrschte, während im Nachgang zu dieser Ersetzung eine technologisch höherwertige Population des modernen Menschen in Europa fortlebte. Auch lagen Funde symbolischer Objekte wie Höhlenmalereien und Schmuck zu jener Zeit umfänglich diesseits jenes Ersetzungsprozesses. Die geradezu offensichtliche Schlussfolgerung bestand damit in

39 Knapp anzumerken ist hier womöglich, dass sich Nordamerika insbesondere im 20. Jahrhundert ebenfalls als bedeutendes politisches, wirtschaftliches und freilich wissenschaftliches Zentrum etablieren konnte. Da dort jedoch zu keinem Zeitpunkt der Menschwerdung archaischen Menschenarten existierten, gelten die vorliegenden Aussagen dennoch uneingeschränkt. Auch fanden asiatische Hochkulturen zwar seit Jahrhunderten Fossilfunde im Rahmen ihrer jeweiligen Ausbeutung natürlicher Bodenschätze und der Schaffung von Infrastruktur, jedoch ohne den Hintergrund der sich in Europa ausbildenden Naturwissenschaften einschließlich der Taxonomien und Evolutionstheorien der Neuzeit.

40 Wie ebenfalls in jenem Kapitel angemerkt wurde gab es sicherlich eine genetische Durchmischung. Dabei verbleibt jedoch unklar, ob es nicht zugleich zu einer Verdrängung des Neandertalers durch den anatomisch modernen Menschen kam und welche Form eine derartige Verdrängung angenommen hat. Hierbei könnte es sich um eine rein ökologisch bzw. demographisch begründete Ersetzung gehandelt haben, jedoch kann durchaus nicht ausgeschlossen werden, dass keine direkten Konfliktsituationen aufgetreten sind. 
einem kulturell und technologisch überlegenen modernen Menschen, welcher den unterlegenen Neandertaler verdrängte.

Auf Basis der Feststellung, dass ein solches Gefälle hinsichtlich Technologien und symbolischem Verhalten plausibel über den Ursprung von Sprachfähigkeit erklärt werden kann, bestand die klassische Auslegung der Datenlage in der Unterstellung einer kognitiven Revolution im anatomisch modernen Menschen vor etwa 50.000 Jahren, welche zu Verhaltensmodernität sowie in Folge zur raschen und unaufhaltsamen Ausbreitung über die gesamte Welt führte (vgl. Shea 2011 für eine einflussreiche Kritik an dieser klassischen Auslegung sowie gegenüber des Konzepts der Verhaltensmodernität). Diese Interpretation dominierte die einschlägige Forschung über Jahrzehnte - ein Umstand, welcher den Widerstand gegenüber der wachsenden jüngeren Fundlage wenigstens teilweise erklärt - und wird bis heute umfänglich von paläoanthropologisch nicht ausreichend informierten Sprachursprungsforschern vertreten.

\subsection{Der sich wandelnde paläoanthropologische Konsens}

Innerhalb der Fundlage der jüngeren Forschung wurden im Kontrast zu jener Interpretation zwei Dinge zunehmend klarer: Erstens zeigt sich vermehrt, dass es keine kognitive Revolution gegeben zu haben scheint, und zweitens finden sich angeblich auf $H$. sapiens beschränkte moderne Verhaltensweisen auch beim Neandertaler. In diesem Sinne legt die jüngere Fundlage entgegen jener klassischen Auffassung sowohl beim anatomisch modernen Menschen als auch beim Neandertaler vermehrt einen inkrementellen, kumulativen Prozess zunehmender Technisierung und Symbolisierung nahe, welcher wiederkehrend lokale Innovationen und lokalen Verlust in einer komplexen kulturellen Dynamik aufweist. Viele Technologien wie Wurfwaffen, Steinklingen und hierarchische, mehrstufige Werkzeuge zeigen sich mit bis zu einer halben Million Jahre als zehnfach älter als zunächst angenommen und eine systematische Verwendung von Farbe etabliert sich ab vor grob 300.000 Jahren und damit bereits vor dem Aufkommen des anatomisch modernen Menschen (vgl. Thieme 1997, Barham 2002, Roebroeks \& Villa 2011, Roebroeks et al. 2012 sowie Muller \& Clarkson 2016).

In diesem Sinne heißt es beispielsweise bei Muller \& Clarkson (2012: 2): „[I]t is now clear that technological evolution is far from linear, but is instead multidirectional, branching and recursive.“ Die soeben erwähnte Klingentechnologie ist dabei „securely dated to well before the Upper Palaeolithic, is not confined to anatomically modern humans, and appears and disappears in many regions over time.“ Zu erwähnen ist dabei trotz der exemplarischen Natur der Erwäh- 
nung von Klingentechnologie die Wichtigkeit derselben für die Paläoanthropologie. So heißt es bei Shea (2011): „In Europe, evidence for the systematic production of prismatic blades coincides with the first appearance of $H$. sapiens in Upper Paleolithic contexts. For decades, Paleolithic archaeologists have routinely inferred a causal connection from this association.“ In anderen Worten bestand einer der gewichtigsten Eckpfeiler der Interpretation einer kognitiven Revolution und der klaren Überlegenheit des anatomisch modernen Menschen im scheinbaren Aufkommen ebendieser Klingentechnologie im Kontext der Ersetzung des Neandertalers durch $H$. sapiens. Dies steht jedoch in einem Konflikt mit den jüngeren Erkenntnisse sowie der vorliegenden Erläuterungen, welche die klassische Auslegung kontrastieren und relativieren.

Ein weiterer höchst bedeutender Eckpfeiler für die Inferenz kognitiver Modernität besteht im symbolischen Verhalten von Frühmenschen. Dieser Aspekt wurde im Unterkapitel zur Menschwerdung ausreichend diskutiert und dort mit zahlreichen Literaturverweisen versehen, sodass hier auf eine ausgiebige Auflistung bzw. Erläuterung verzichtet werden kann. Es bleibt dennoch zusammenfassend anzumerken, dass Körperschmuck und ähnliche Gegenstände in zunehmend großem Umfang dokumentiert werden. Entsprechende Verhaltensweisen nahmen an Intensität und Komplexität anscheinen über Zeit zu und finden sich in vergleichbarem Ausmaß sowohl im anatomisch modernen Menschen als auch im Neandertaler. Es zeigt sich somit anhand jener und weiterer Aspekte, dass die frühere Interpretation des klar überlegenen modernen Menschen ein Datenartefakt darstellt, denn zweifelsohne sind in einer kumulativen Kultur spätere Zeugnisse den früheren überlegen, sodass der Vergleich von Zeugnissen von vor der Ersetzung mit Zeugnissen von nach der Ersetzung ein verfälschendes Bild liefern. Wenn stattdessen das Verhalten der beiden Menschenarten zu parallelen Zeitpunkten vor der Ersetzung verglichen wird, so löst sich der Eindruck der klassischen Interpretation weitestgehend auf (vgl. nochmals Finlayson et al. 2012, Dediu \& Levinson 2013 und 2018, Villa \& Roebroeks 2014, Roebroeks \& Soressi 2016 sowie Hoffecker 2018 und nicht zuletzt Shea 2011 inklusive der Einführung seines einflussreichen Konzepts der Verhaltensvariabilität im Kontrast zur Verhaltensmodernität, welche die kulturelle Kontinuität seit $H$. heidelbergensis noch einmal zusätzlich hervorzuheben vermag).

Klärend soll analog zu Ausführungen im einführenden paläoanthropologischen Kapitel nochmals angemerkt werden, dass innerhalb der einschlägigen Fundlage durchaus der Eindruck höherer Intensität hinsichtlich dieser Verhaltensweisen seitens des anatomisch modernen Menschen naheliegt, jedoch lässt sich dies potenziell auf zweierlei Weise erklären, anstatt sogleich eine kognitive oder kulturelle Ungleichheit zu postulieren. Dies geschieht erstens darüber, 
dass der Neandertaler unter vergleichsweise ungünstigen klimatischen Bedingungen zu leben hatte, womit mehr Arbeitsaufwand in ein bloßes Überleben zu investieren waren, und zweitens anhand der im anatomisch modernen Menschen wesentlich stärker ausgeprägten Populationsdynamik, welche eine höhere kulturelle Stabilität und leistungsfähigere kulturelle Akkumulation ermöglicht. In anderen Worten waren die Neandertaler-Populationen, wenn verglichen mit dem anatomisch modernen Menschen derselben Zeit, gegenüber einander isoliert, hatten eine geringe Bevölkerungsdichte und insgesamt kleine Populationsgrößen (möglicherweise bis zu zehnfach kleiner als beim anatomisch modernen Menschen; vgl. nochmals die Ausführungen im Unterkapitel zur Menschwerdung einschließlich der Autoren Prüfer et al. 2014, Castellano et al. 2014, Mafessoni \& Prüfer 2017 sowie Vaesen et al. 2019).

\subsection{Zum Ausmaß der kognitiven und kulturellen Modernität des Neandertalers}

Des Weiteren existieren mehrere Argumente, eine kognitive Vergleichbarkeit zwischen dem Neandertaler und dem zeitgenössischen anatomisch modernen Menschen anzunehmen. Dies schon einmal aufgrund der qualitativen Vergleichbarkeit der Zeugnisse der beiden Arten, wie beispielsweise die eingangs zitierten Dediu \& Levinson (2018) argumentieren. Weiterhin wird in der einschlägigen Literatur inzwischen die Position angenommen, dass bereits $H$. heidelbergensis als ein Taxon, das entweder in oder nahe bei der Trennung der Linien zum Neandertaler respektive dem anatomisch modernen Menschen zu verorten ist, in kognitiver sowie physiologischer Hinsicht die Grundlage für jene späteren Entwicklungen liefert. Dies zeigt sich an Untersuchungen von Fossilien zur oralen Befähigung dieser Art, welche modern oder wenigstens beinahe modern zu sein scheint (Martínez et al. 2004 und 2013), einem beinahe modernen Gehirnvolumen (Buck \& Stringer 2014), dem kulturellen kumulativen Kontinuum ab dieser Art bis zum Neandertaler respektive dem anatomisch modernen Menschen (Powell, Shennan \& Thomas 2009, Shea 2011, Eren \& Lycett 2012, Muller \& Clarkson 2016 sowie Hosfield \& Cole 2018) und nicht zuletzt an genetischen Fundamenten wie der modernen menschlichen Variante des FOXP2Gens, welche in den $H$. heidelbergensis nachfolgenden Arten umfänglich nachgewiesen wurde (Krause et al. 2007, Reich et al. 2010 sowie Paixáo-Côrtez et al. 2012) und welche daher mit größter Wahrscheinlichkeit bereits in H. heidelbergensis fixiert war. Erklärendes zum FOXP2-Gen folgt im direkt nachfolgenden Analysekapitel, in welchem dessen Forschungsgeschichte sowie dessen Rolle innerhalb der Sprachursprungsforschung diskutiert werden soll. 
$\mathrm{Zu}$ diesen Feststellungen gesellt sich die ebenfalls junge Erkenntnis, dass der Neandertaler - insbesondere innerhalb jenes Ersetzungskontexts vor grob 40.000 Jahren - vermutlich durchaus in engen sozialen Kontakt mit dem in Europa eindringenden anatomisch modernen Menschen trat und dass er dabei möglicherweise kulturelle und technologische Verhaltensstrategien an den anatomisch modernen Menschen weitergab, denn eine Reihe von Verhaltensweisen begannen im Neandertaler bereits vor dem Ersetzungsprozess und bilden trotz der Ersetzung des Neandertalers anscheinend ein Kontinuum mit dem nachfolgenden anatomisch modernen Menschen (Tostevin 2007, Soressi et al. 2013, Finlayson \& Finlayson 2016). Besonders im Nachgang zu dieser Feststellung besteht ein weiteres zentrales Argument schließlich im ökologischdemographischen Gegendruck, den der Neandertaler anscheinend lange Zeit trotz wiederholter Kontaktphänomene aufrechterhalten hat.

Wenn der anatomisch moderne Mensch dem Neandertaler schlichtweg überlegen gewesen wäre, so läge nahe, dass eine entsprechende Verdrängung früher hätte geschehen sollen. Ein unregelmäßiger Kontakt zwischen den beiden Arten bzw. ihren jeweiligen archaischen Frühformen ist für den Zeitraum zwischen vor 460.000 Jahren und 219.000 Jahren anhand genetischer Daten belegt (Posth et al. 2017) und Fossilfunde des anatomisch modernen Menschen datieren in Griechenland zurück auf bis zu 210.000 Jahre (Harvati et al. 2019). Dennoch hielt sich der Neandertaler über diesen Gesamten Zeitraum nicht nur als die in Europa dominierende Art, sondern ersetzte den anatomisch modernen Menschen in bereits besiedelten Gebieten in Südosteuropa und dem Nahen Osten wiederkehrend. Auch als der anatomisch moderne Mensch schließlich ab vor 130.000 Jahren in mehreren Auswanderungswellen nach Asien vorstieß und wenige zehntausend Jahre später China und Australien besiedelte (Liu et al. 2015, Clarkson et al. 2017, Groucutt et al. 2015 sowie Cabrera et al. 2018), verblieb der Neandertaler die einzige menschliche Population in Europa und Westasien.

Ein generelles Muster prähistorischer Populationen in Europa sowohl vor als auch nach der Ersetzung des Neandertalers bestand darin, dass Kaltzeiten zu einer teilweisen Entvölkerung des Kontinents führten, bevor darauf folgende Warmzeiten eine Ausbreitung wieder ermöglichten. So lebten sowohl H. heidelbergensis als auch der Neandertaler zeitweise in England (Stringer et al. 1998, Streeter et al. 2001, Bates et al. 2013, Stout et al. 2014) und auch der moderne Mensch besiedelte bzw. verließ England wiederholt, bevor erst vor etwa 12.000 Jahren eine kontinuierliche Population etabliert werden konnte (Pettitt \& White 2012). Paläoklimatische Untersuchungen legen dabei nahe, dass das Timing eines ebensolchen Zyklus zur nachfolgenden Ersetzung des Neandertaler zu 
passen scheint (Alcaraz-Castano 2017, Wolf et al. 2018, Melchionna et al. 2018, Staubwasser et al. 2018) und damit im Kontext des ohnehin bereits bestehenden demographischen Ungleichgewichts (vgl. nochmals Prüfer et al. 2014, Castellano et al. 2014, Mafessoni \& Prüfer 2017 sowie Vaesen et al. 2019) die relativ plötzliche Ersetzung fundiert haben könnte. Eine zusätzliche Schwächung der Neandertaler-Population könnte in einem derartigen Szenario durch den Ausbruch eines Supervulkans in Süditalien vor etwas mehr als 39.000 Jahren geschehen sein (De Vivo et al. 2001, Costa et al. 2012) und auch die Schwächung der Fitness des Neandertaler-Genoms durch die langfristig geringe Populationsgröße muss innerhalb der vorliegenden Interpretation bedacht werden (u.a. Vaesen et al. 2019).

Aus all dem folgt ein Gesamtbild, in welchem eine quasi moderne Variabilität des Verhaltens im gemeinsamen Formenkreis um $H$. sapiens umfänglich zu existieren scheint und sich insbesondere im Neandertaler in zum anatomisch modernen Menschen vergleichbaren Verhaltensweisen manifestiert. Wenn sich dieser Anschein im Forschungsverlauf weiter erhärten sollte, so bestünde seit $H$. heidelbergensis bzw. ihm naheliegenden Taxa eine nennenswerte Kontinuität hin zu den nachfolgenden Schwesterngruppen, also des Neandertalers und des anatomisch modernen Menschen. Innerhalb dieser kontinuierlichen Entwicklung findet sich einerseits aufgrund demographisch-ökologischer Beschränkungen eine komplexe Dynamik kultureller Errungenschaft und kulturellen Verlusts, jedoch andererseits nichtsdestotrotz eine Akkumulation geistigkultureller Reife, die sich anhand des einsetzenden und sich dann verstärkenden symbolischen Verhaltens offenbart. Eine ungleiche Populationsdynamik und für den Neandertaler unglückliche ökologische Bedingungen führen schließlich zur Ersetzung desselben durch den anatomisch modernen Menschen, ohne dass eine qualitative Dominanz des modernen Menschen in kognitiven, technologischen oder sprachlichen Domänen unterstellt werden muss.

Dieses Szenario wird durch einen Hinweis auf die globale Populationsdynamik des modernen Menschen lange nach der Ersetzung des Neandertalers weiter unterstrichen, denn diese ist von vergleichbaren demographisch begründeten Ersetzungen bzw. Verdrängungen aufgrund Expansion oder Wanderung von Populationen durchsetzt. Faktoren kumulativer kultureller Phänomene spielen auch dort eine zentrale Rolle und führen mitunter zu plötzlichen demographischen Ersetzungen (vgl. Ghirlanda \& Enquist 2007, Malmstöm et al. 2009, Skoglund et al. 2012, Aoki 2015, Hofmanová et al. 2016 sowie Goldberg et al. 2017 für derartige Ersetzungen im prähistorischen Europa). Auch die europäische Kolonisierung Amerikas würde heute niemals als Zeichen dafür gedeutet, dass Europäer kognitiv oder auf sonstige Weise biologisch überlegen gewesen 
wären, und indigene Völker, welche noch heute technologische Innovationen oder symbolische Zeugnisse, die Teil des Repertoires des Neandertalers waren, missen, sind dennoch sprachlich sowie kognitiv vollwertige Mitglieder unserer Art. Zuletzt finden sich derartige Ersetzungen bereits zwischen Populationen des Neandertalers, sodass vollends klar wird, dass eine Ersetzung einer menschlichen Population durch eine andere innerhalb der Spätphase menschlicher Evolution nicht automatisch eine kognitiv-kulturelle respektive sprachliche Ungleichheit impliziert (vgl. das Review von Gokcumen 2019).

Die allgemeine Schlussfolgerung besteht damit darin, dass Sprache innerhalb jenes Formenkreises ein ubiquitäres Phänomen gewesen sein könnte und insbesondere hinsichtlich des Neandertalers mit nennenswerter Plausibilität vermutet werden kann. Klärend muss angemerkt werden, dass aufgrund der Natur der indirekten Rekonstruktion nicht mit Sicherheit auf jenes Urteil geschlossen werden kann und dass in Folge Sprache in letzter Konsequenz durchaus jünger sein könnte als dort suggeriert. Es verbleibt jedoch in Anbetracht der umfassenden indirekten Triangulation innerhalb des soeben Erläuterten zuzugestehen, dass es für eine derartige zweifelnde Annahme keine gleichwertig überzeugende Begründung zu geben scheint. Die Konklusion einer wahrscheinlichen Verwendung von Sprache beim Neandertaler sowie einer allgemein alten Sprachbefähigung im Formenkreis ist damit als innerhalb der aktuellen Forschungslage wahrscheinlich anzusehen.

Abschließend muss noch die Verbindung mit dem bereits vorgestellten Methodenkatalog hergestellt werden, denn dieser fundiert die Rezeption, Synthese und Argumentation der obigen Ausführungen sowie der darin enthaltenen Literaturverweise. Dabei hat sich zwar im Sinne von M1 noch kein abschließender Konsens herausbilden können, jedoch wurde dieser Umstand anhand der Forschungsgeschichte ausreichend begründet. Ansonsten ist nach M3 sicherlich primär die paläoanthropologische Expertenposition zu priorisieren und ebendiese bewegt sich jüngst in Richtung des vorgestellten Szenarios. Zugleich repliziert sich dieses Szenario mit steigender Frequenz innerhalb der einschlägigen Literatur (M5). Ebendiese gerichtete Entwicklung der Literatur erlaubt für sich wiederum ein Urteil zur Validität (M8) insbesondere im Kontext eines technologisch und methodisch zunehmend leistungsfähigen Forschungsfeldes (M9) - und auch wenn nach M1 und M10 noch keine vollkommen geklärte Situation geschaffen werden konnte, so muss hinsichtlich M11 aufgrund vorgenannter Prinzipien sowie der Feststellung, dass die Gegenposition vermehrt auf die Anwendung von in M12 genannten Problemen zurückweichen muss, zugunsten der jüngeren Ergebnisse und Interpretation entschieden werden. 